\title{
O Uso de Listas de Verificação para Avaliação da Usabilidade de um Ambiente de EAD na Web
}

\author{
Joelson Piaia ${ }^{1}$, Isabela Gasparini ${ }^{1}$ \\ ${ }^{1}$ Universidade do Estado de Santa Catarina \\ joelsonpiaia@yahoo.com.br, isabela@joinville.udesc.br
}

\begin{abstract}
Resumo. A utilização da internet como meio para a Educação a Distância possibilita muitas vantagens em relação aos métodos tradicionais. Contudo para que as potencialidades das novas tecnologias se revertam em benefícios para os usuários é necessário que os sistemas tenham usabilidade. Neste artigo são apresentados os resultados obtidos com a avaliação de usabilidade do ambiente aluno do AdaptWeb. A avaliação foi realizada através da aplicação de duas listas de verificação; uma genérica e outra voltada a ambiente de educacionais.
\end{abstract}

\section{Introdução}

A Educação a Distância (EAD) tem reavivado suas práticas e procurado se adaptar as possibilidades das novas tecnologias. As vantagens da internet como a flexibilidade do tempo, quebra de barreiras espaciais e transmissão instantânea de materiais, tornam-se necessidades do mundo moderno.

No cenário atual muito tem se falado sobre a usabilidade. Observa-se cada vez mais a necessidade de se criar softwares que permitam aos usuários atingirem seus objetivos de maneira eficaz, eficiente e agradável. Para garantir esses objetivos, é imprescindível a usabilidade seja avaliada. Várias técnicas podem ser utilizadas para este fim, dentre elas estão as listas de verificação, também conhecidos por checklist. As listas de verificação constituem uma maneira rápida e fácil de obter um diagnóstico geral sobre a usabilidade do sistema avaliado.

Este artigo apresenta a comparação dos resultados obtidos com a utilização de duas listas de verificação de usabilidade em um ambiente EAD via Web. O ambiente escolhido para a avaliação foi o AdaptWeb (Ambiente de Ensino Aprendizagem Adaptativo via Web), um ambiente cuja finalidade é de disponibilizar um mesmo conteúdo adaptado conforme o perfil de usuário.

A seção 2 deste artigo apresenta o ambiente de EAD AdaptWeb, onde as listas de verificação foram aplicadas. A seção 3 comenta sobre o conceito de usabilidade e sobre as técnicas de avaliação. A seção 4 explica as avaliações realizadas no ambiente. A seção 5 faz a análise e comparação dos resultados. Por fim, a seção 6 apresenta as considerações finais do artigo. 


\section{AdaptWeb}

A Educação a Distância (especialmente via web) permite integrar múltiplas mídias, linguagens e recursos, organizar a informação apresentada das mais diversas maneiras, criar interações entre as pessoas e os objetos de conhecimento além de elaborar e compartilhar conhecimento. O conteúdo pode ser apresentado em várias mídias, tais como: hipertextos, imagens e vídeos (ALMEIDA, 2003).

O ambiente escolhido para a avaliação neste trabalho foi o AdaptWeb (Ambiente de Ensino-Aprendizagem Adaptativo na Web). Um ambiente de EAD hipermídia adaptativo para web, desenvolvido através de uma parceria da UFRGS (Universidade Federal do Rio Grande do Sul) com a UEL (Universidade Estadual de Londrina) e colaboração do CNPq. Este sistema tem por objetivo adaptar o conteúdo, a apresentação e a navegação de acordo com o modelo de usuário, disponibilizando um mesmo conteúdo adaptado para estudantes de grupos distintos (GASPARINI, 2003).

A interface do ambiente aluno, onde são apresentados os conteúdos definidos na fase de autoria, foi projetada visando obter simplicidade e consistência. Este ambiente utiliza a informação referente à formação do aluno para adaptar a navegação dentro dos conceitos, visto que um grupo de usuários pode acessar determinados conceitos, exercícios e exemplos e outro não. O AdaptWeb armazena também informações referentes ao conhecimento que o aluno já adquiriu no ambiente e utiliza tais informações para prover a adaptabilidade, não deixando que um aluno acesse determinado conceito se ainda não estudou os pré-requisitos (GASPARINI, 2003).

\section{Usabilidade}

Conforme a Norma ISO 9241-11 a usabilidade é definida como a capacidade que um sistema interativo oferece ao seu usuário para a realização de tarefas, de maneira eficaz, eficiente e agradável, em um determinado contexto de operação (NBR 9241-11, 2002). Nielsen define usabilidade de acordo com cinco atributos: facilidade de aprendizagem; eficiência; facilidade de memorização; recuperação de erros; satisfação do usuário (NIELSEN, 1993).

Os usuários de sistemas não estão interessados em detalhes técnicos do software, para eles o software é a interface, ou seja, segundo sua visão a qualidade da interface e da interação determinam a qualidade do sistema. Interfaces bem projetadas auxiliam o usuário no aumento de produtividade e os desenvolvedores com diminuição de custos com suporte técnico. Em contrapartida, problemas de usabilidade trazem uma série de condições adversas, dentre elas: requerem treinamento excessivo; desmotivam a exploração; confundem o usuário; induzem ao erro; geram insatisfação; diminuem a produtividade e não trazem o retorno do investimento previsto (PRATES; BARBOSA, 2003).

\subsection{Avaliação de Usabilidade}

Avaliar a usabilidade é importante, pois, através das avaliações são detectados problemas e oportunidades de melhoria na interação (NBR 9241-11, 2002). Existem 
vários métodos de avaliação de interfaces, diferindo entre si em diversos aspectos, como a fase do projeto em que podem ou devem ser aplicados, a maneira como os dados são coletados, os tipos de dados coletados e o tipo de análise realizada (PRATES; BARBOSA, 2003).

Podem-se distinguir três tipos de técnicas para avaliação de usabilidade. As Técnicas Preditivas são utilizadas para avaliar a usabilidade de um software sem a participação direta do usuário; os avaliadores, em geral, são especialistas em usabilidade que utilizam seu conhecimento, experiência e listas de critérios. Algumas das técnicas preditivas, no entanto, podem ser aplicadas por especialistas no domínio ou mesmo pelos projetistas do ambiente. As Técnicas Objetivas ou Empíricas contam com a participação direta do usuário, onde a interação entre usuário e sistema é observada. Por fim, as Técnicas Prospectivas utilizam entrevistas e questionários para buscar a opinião dos usuários sobre a interação com o sistema (CYBIS, 2003). A Figura 1 mostra um esquema com exemplos de cada grupo de técnicas.

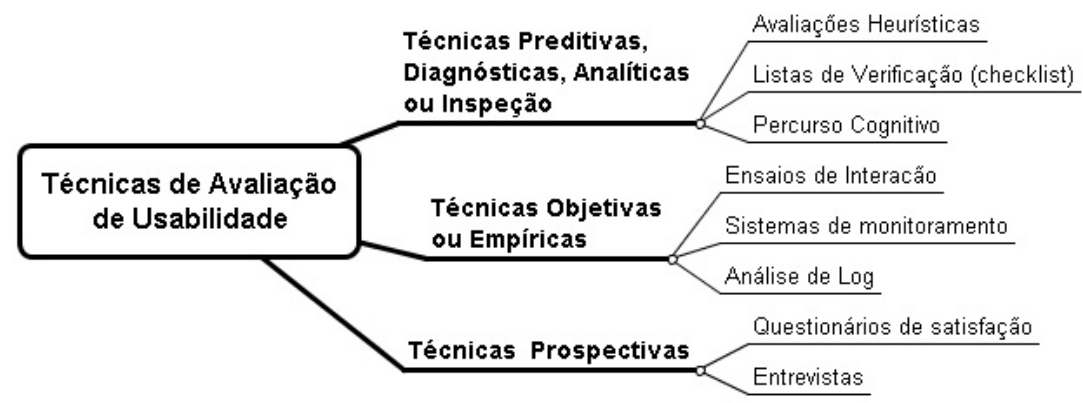

Figura 1 - Técnicas de Avaliação de Usabilidade (Adaptado: CYBIS, 2003)

Neste trabalho a opção por utilizar Listas de Verificação foi tomada, pois as permitem obter resultados rápidos sem a necessidade de avaliação por especialistas em IHC (Interação Humano-Computador).

\subsection{Listas de Verificação (checklists)}

As listas de verificação não são necessariamente direcionadas aos especialistas em usabilidade, podendo ser utilizadas por outros profissionais, tais como programadores e analistas (BARROS, 2003). Nas inspeções via listas de verificação a qualidade da própria lista determina a efetividade da avaliação, visto que o conhecimento de IHC está embutido na listas de verificação. Listas bem elaboradas produzem resultados mais uniformes e abrangentes, identificando grande parte dos problemas de usabilidade (CYBIS, 2003).

Para verificar a usabilidade do ambiente aluno do AdaptWeb foram aplicadas duas listas de verificação: o ErgoList, desenvolvido pelo Laboratório de Utilizabilidade da Universidade Federal de Santa Catarina (LabiUtil) disponibilizado na web e uma Lista de Verificação Proposta por Dringus e Cohen (DRIGUS; COHEN, 2005) (ERGOLIST, 2006). 


\subsubsection{ErgoList}

O ErgoList é um checklist para avaliação autônoma da usabilidade de interfaces humano-computador baseado nos Critérios Ergonômicos de Scapin e Bastien (BASTIEN; SCAPIN, 1993). É composto por três módulos: Checklist; Questões e Recomendações. O módulo Checklist ajuda a realizar a inspeção sistemática da interface. As questões estão divididas em 18 critérios. Em qualquer ponto do checklist é possível acionar um glossário obtendo assim mais informações e exemplos que ilustram cada questão. O módulo Questões permite ao avaliador conhecer informalmente as questões que compõe o ErgoList. No módulo Recomendações são apresentadas recomendações gerais para melhoria da interface. A opção por pelo ErgoList se deu pelo fato de ser uma lista bem conhecida pela comunidade de IHC e bastante utilizada além de possuir uma ferramenta disponível na internet para ajudar na avaliação.

\subsubsection{Lista de Verificação Proposta por Dringus e Cohen}

Este checklist, baseado nas heurísticas de usabilidade de Nielsen (NIELSEN, 2003), visa avaliar a usabilidade de sistemas de EAD. Para elaborar este checklist as autoras fizeram inicialmente uma avaliação heurística no sistema WebCT. Os problemas encontrados foram organizados e separados em 13 heurísticas. O resultado obtido é uma lista de verificação adaptada, em seu estágio de esboço (DRIGUS; COHEN, 2005). Esta lista de verificação foi escolhida por ser uma publicação nova e específica para ambientes de EAD, além de ser baseada nas heurísticas de Nielsen, o que difere do ErgoList que é baseado nos critérios ergonômicos.

\section{Aplicação das Listas de Verificação no Ambiente Aluno do AdaptWeb}

A aplicação das listas de verificação no AdaptWeb foi realizada na versão 0.9.2 beta, instalada no servidor da UEL. Toda a interface que o aluno pode utilizar no ambiente foi avaliada, desde o login até o ambiente de ensino/aprendizado onde as disciplinas são apresentadas. No lado cliente foi utilizado um microcomputador com sistema operacional Windows XP, navegador Internet Explorer 6 SP2, conexão com a internet de banda larga e monitor configurado em 1024 x 768 pixels. Antes de iniciar as avaliações o avaliador fez um reconhecimento e navegação pelo ambiente.

\subsection{Avaliação com o ErgoList}

A primeira avaliação realizada no AdaptWeb foi com o Ergolist, utilizando o site disponível na web. A aplicação da técnica e registro das respostas durou 9 horas e foi realizada com intervalos entre cada critério avaliado. Cada questão foi verificada quanto a sua aplicabilidade no ambiente. Para as questões aplicáveis sua conformidade foi avaliada. Na Tabela 1 é apresentado um resumo dos resultados obtidos com a aplicação do ErgoList. Várias questões (25\%) foram consideradas não aplicáveis no ambiente, tais questões se referiam a aspectos de interface não utilizados no AdaptWeb.

Dois pontos muito positivos observado no ErgoList são o glossário e os comentário sobre cada questão; estes recursos facilitaram o entendimento da técnica, 
pois qualquer dúvida quanto aos termos ou questões pode ser rapidamente resolvida através da consulta ao glossário.

Tabela 1 - Resumo dos resultados obtidos com o ErgoList

\begin{tabular}{c|c|c|c|c}
\hline Critério & $\begin{array}{c}\text { Total de } \\
\text { Questões }\end{array}$ & $\begin{array}{c}\text { Questões Não } \\
\text { Aplicáveis }\end{array}$ & $\begin{array}{c}\text { Questões } \\
\text { Conformes }\end{array}$ & $\begin{array}{c}\text { Questões Não } \\
\text { conformes }\end{array}$ \\
\hline Presteza & 17 & 5 & 8 & 4 \\
\hline Agrupamento por localização & 11 & 2 & 8 & 1 \\
\hline Agrupamento por formato & 17 & 2 & 12 & 3 \\
\hline Feedback & 12 & 0 & 11 & 1 \\
\hline Legibilidade & 27 & 6 & 20 & 1 \\
\hline Concisão & 14 & 7 & 7 & 0 \\
\hline Ações Mínimas & 5 & 0 & 3 & 2 \\
\hline Densidade informacional & 9 & 2 & 6 & 1 \\
\hline Ações explicitas & 4 & 0 & 4 & 0 \\
\hline Controle do Usuário & 4 & 0 & 3 & 1 \\
\hline Flexibilidade & 3 & 0 & 1 & 2 \\
\hline Experiência do Usuário & 6 & 2 & 4 & 0 \\
\hline Proteção contra erros & 7 & 6 & 1 & 0 \\
\hline Mensagens de erro & 9 & 1 & 1 & 7 \\
\hline Correção de erros & 5 & 2 & 3 & 0 \\
\hline Consistência & 11 & 0 & 10 & 1 \\
\hline Significados & 12 & 4 & 7 & 1 \\
\hline Compatibilidade & 21 & 9 & 10 & 2 \\
\hline Total & $\mathbf{1 9 4}$ & $\mathbf{4 8}(\mathbf{2 5 \% )}$ & $\mathbf{1 1 9} \mathbf{( 6 1 \% )}$ & $\mathbf{2 7}$ (14\%) \\
\hline
\end{tabular}

\subsection{Avaliação com o Checklist de Dringus e Cohen}

Para aplicar o checklist de Dringus e Cohen, primeiramente foram traduzidas as questões para o português, neste trabalho foram gastas 4 horas. A verificação da conformidade das questões durou 5 horas, onde cada questão foi lida e avaliadas quanto a sua aplicabilidade. Para as questões aplicáveis sua conformidade foi avaliada. Este checklist não possui ferramenta eletrônica, portanto as questões foram respondidas em uma planilha. A Tabela 2 mostra um resumo dos resultados obtidos com aplicação do checklist de Dringus e Cohen. Apesar de ser voltado para sistemas de EAD foi observado um grande índice de questões não aplicáveis, isto se deve a dois fatores: muitas questões deste checklist são relativas a ambientes de autoria, não avaliados neste trabalho; um grande número de questões foram descartadas pois tratam de aspectos não contemplados pelo AdaptWeb mas que geralmente são encontrados em ambientes de EAD, como por exemplo a comunicação e colaboração entre alunos e professores ou alunos e alunos. 
Tabela 2 - Resumo dos resultados obtidos com o checklist de Dringus e Cohen

\begin{tabular}{c|c|c|c|c}
\hline Heurística & $\begin{array}{c}\text { Total de } \\
\text { Questões }\end{array}$ & $\begin{array}{c}\text { Questões Não } \\
\text { Aplicáveis }\end{array}$ & $\begin{array}{c}\text { Questões } \\
\text { Conformes }\end{array}$ & $\begin{array}{c}\text { Questões Não } \\
\text { conformes }\end{array}$ \\
\hline Visibilidade & 30 & 6 & 19 & 5 \\
\hline Funcionalidade & 8 & 2 & 6 & 0 \\
\hline Estética & 15 & 0 & 14 & 1 \\
\hline Feedback e ajuda & 10 & 0 & 9 & 1 \\
\hline Prevenção de erros & 7 & 3 & 0 & 4 \\
\hline Facilidade de lembrança & 9 & 4 & 5 & 0 \\
\hline Gerenciamento do curso & 16 & 16 & 0 & 0 \\
\hline Interatividade & 28 & 28 & 0 & 0 \\
\hline Flexibilidade & 13 & 8 & 1 & 4 \\
\hline Consistência & 9 & 0 & 8 & 1 \\
\hline Eficiência & 4 & 0 & 4 & 0 \\
\hline Redução de redundância & 3 & 2 & 1 & 0 \\
\hline Acessibilidade & 9 & 2 & 6 & 1 \\
\hline Total & $\mathbf{1 6 1}$ & $\mathbf{7 1 ~ ( 4 4 \% )}$ & $\mathbf{7 3 ~ ( 4 5 \% )}$ & $\mathbf{1 7}$ (11\%)
\end{tabular}

Foi observado que esta lista de verificação possui questões abertas e abrangentes, o que dificulta a avaliação. Freqüentemente são utilizados termos técnicos e não existe um manual para o entendimento das questões. Outra dificuldade na utilização desta lista de verificação foi a existência de questões inversas, onde uma resposta negativa na questão representa uma conformidade da interface, exigindo do avaliador conhecimentos de usabilidade para avaliar a conformidade ou não da questão.

\section{Análise dos Resultados}

Com a aplicação das duas listas de verificação foram identificadas 44 questões não conformes. Destacando as sobreposições, 16 não conformidades foram identificadas apenas pelo ErgoList e 11 apenas pelo checklist de Dringus e Cohen; 11 questões do ErgoList apontaram os mesmos problemas que 6 questões do checklist de Dringus e Cohen. A Figura 2 apresenta a relação de questões não conformes entre os cheklists.

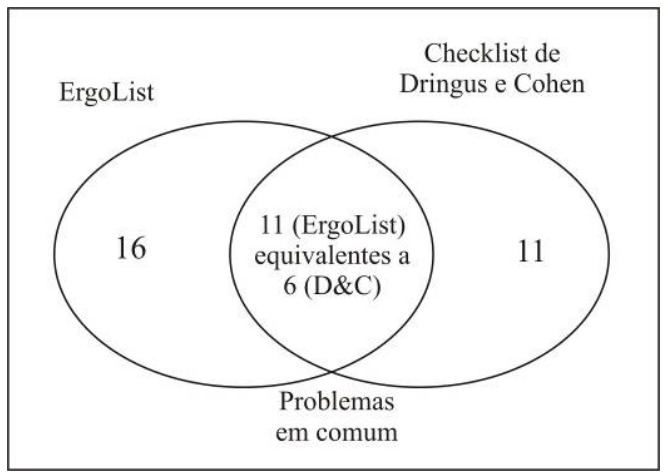

Figura 2 - Número de questões não conformes comuns

Todas as respostas não conformes coletadas durante a avaliação receberam classificação quanto a sua natureza, tipo de tarefa e tipo de usuário (CYBIS, 2003). Ainda, as questões que foram respondidas como não conformes e que não causam prejuízo ao usuário, foram classificadas como problemas falsos, ficando isentas das demais classificações. 
A Tabela 3 apresenta a comparação dos resultados obtidos nas duas listas de verificação, classificadas quanto a sua natureza. Ao todo, 11 problemas foram considerados como falsos, ou seja, aspectos da interface que embora identificados como problemas pelo método de avaliação não causam diminuição do tempo de execução das tarefas, mas nem por isso devem ser descartados. A correção destes problemas pode melhorar a percepção subjetiva do usuário tornando o sistema mais agradável de ser utilizado. Ainda pode ser possível trazer melhorias ao ambiente através da implementação de características ainda não disponíveis no AdaptWeb. Descartando-se os problemas falsos, 33 questões foram consideradas não conformes. Os problemas classificados como barreiras se referem a aspectos da interface em que o usuário esbarra e não aprende a suplantá-los, já os obstáculos são problema que os usuários aprendem a suplantar, enquanto os ruídos constituem problemas que não se caracterizam como barreira ou obstáculo mas mesmo assim causam diminuição do desempenho na realização das tarefas.

Tabela 1 - Comparação das respostas não conformes quanto à natureza

\begin{tabular}{c|c|c|c|c|c}
\hline Lista de Verificação & Barreira & Obstáculo & Ruído & Falso & Total \\
\hline ErgoList & 7 & 7 & 7 & 6 & 27 \\
\hline Checklist D\&C & 6 & 0 & 6 & 5 & 17 \\
\hline Total Geral & 13 & 7 & 13 & 11 & 44 \\
\hline
\end{tabular}

Para as classificações seguintes foram descartados os problemas considerados como falsos, sendo computados os problemas classificados como barreiras, obstáculos e ruídos. A Tabela 4 mostra a comparação das respostas não conformes quanto ao tipo de tarefa. A maioria dos problemas encontrados pelas duas listas de verificação foram classificados como principais, ou seja, a maioria dos problemas identificados na avaliação afetam tarefas principais ou freqüentes do ambiente. Os problemas secundários afetam tarefas pouco freqüentes ou de segunda importância.

Tabela 4 - Comparação das respostas não conformes quanto ao tipo de tarefa

\begin{tabular}{c|c|c|c}
\hline Lista de Verificação & Principal & Secundário & Total \\
\hline ErgoList & 16 & 5 & 21 \\
\hline Checklist D\&C & 8 & 4 & 12 \\
\hline Total & 24 & 9 & 33 \\
\hline
\end{tabular}

A Tabela 5 mostra a comparação das respostas não conformes quanto ao tipo de usuário. A maioria dos problemas apontados pelos dois checklists foram classificados como gerais, ou seja, afetam qualquer tipo usuário. Os problemas de iniciação, que afetam os usuários iniciantes no sistema, somaram 4. O ErgoList ainda apontou um problema que foi classificado como avançado por afetar a interação apenas para usuários experientes no sistema, que necessitam de maior agilidade e 1 problema classificado como especial, por afetar apenas usuários portadores de necessidades especiais.

Tabela 2 - Comparação das respostas não conformes quanto ao tipo de usuários

\begin{tabular}{c|c|c|c|c|c}
\hline Lista de Verificação & Geral & De iniciação & Avançado & Especial & Total \\
\hline ErgoList & 16 & 3 & 1 & 1 & 21 \\
\hline Checklist D\&C & 11 & 1 & 0 & 0 & 12 \\
\hline Total & 27 & 4 & 1 & 1 & 33 \\
\hline
\end{tabular}


As questões não conformes apontaram uma série de problemas tais como: tratamento inadequado das mensagens de erro, limitações no diálogo entre professor e alunos, qualidade da ajuda, continuidade do diálogo, feedback, minimização dos movimentos do cursor, identificação de campos obrigatórios, personalização do sistema, distinção de ícones e falta de padrões no posicionamento de botões em formulários.

O ErgoList mostrou-se mais apto a encontrar falhas pontuais na interface, que constituem violações claras dos critérios ergonômicos. Enquanto o checklist de Dringus e Cohen foi melhor para identificar problemas mais abrangentes ou aspectos comumente encontrados em softwares de EAD que ainda não estão implementados no AdaptWeb.

\section{Considerações Finais}

Este artigo teve como finalidade apresentar e comparar os resultados obtidos na avaliação de usabilidade do ambiente aluno do AdaptWeb via listas de verificação. Para isto, foram utilizados o ErgoList, já bem difundido na comunidade de IHC brasileira e o checklist proposto por Dringus e Cohen, desenvolvido especificamente para ambientes de EAD.

A avaliação com o Ergolist se mostrou mais fácil de ser executada, a ferramenta on-line disponibilizada pelo LabiUtil ajudou muito no processo de avaliação. Eventuais dúvidas quanto às questões puderam ser facilmente sanadas com a ajuda do glossário, sem a necessidade de interromper a avaliação.

A aplicação deste checklist de Dringus \& Cohen, no entanto, foi mais difícil, visto que algumas questões são de difícil interpretação e muito abrangentes. Muitas questões requerem do avaliador conhecimentos de usabilidade para julgar a conformidade, esta particularidade não deveria ocorrer, pois uma lista de verificação deveria ser facilmente utilizada por avaliadores não especialistas em usabilidade (CYBIS, 2003).

O checklist de Dringus \& Cohen se mostrou uma opção muito adequada para softwares de EAD, apontando problemas não tão triviais. Contudo este checklist ainda necessita melhor clareza e padronização das questões. O ErgoList cumpriu bem seu propósito fornecendo uma lista abrangente dos problemas de usabilidade do AdaptWeb.

Os resultados das avaliações com as duas listas de verificação aplicadas podem ser aplicados a fim de melhorar a qualidade da interface do ambiente estudado, bem como servir de complemento ou base para outras técnicas de avaliação aplicadas no ambiente em questão.

\section{Referências}

ALMEIDA, M. E. B. de. Educação a distância na internet: abordagens e contribuições dos ambientes digitais de aprendizagem. Educ. Pesqui, jul./dez. 2003, vol.29, no.2, p.327-340. ISSN 1517-9702.

BASTIEN, J. M. Christian; SCAPIN Dominique L. Ergonomic Criteria for the Evaluation of Human-Computer Interfaces. Technical report $N^{\circ}$ 156. 1993.

CYBIS, W. A. Engenharia de Usabilidade: Uma abordagem ergonômica. LabiUtil, 2003, <http://www.labiutil.inf.ufsc.br>. 
DRINGUS, L. P. COHEN, M. S. An Adaptable Usability Heuristic Checklist for Online Courses. 35th ASEE/IEEE Frontiers in Education Conference. 19-22/10/2005. Indianápolis, IN.

ERGOLIST. Disponível em: <http://www.labiutil.inf.ufsc.br/ergolist/>.

GASPARINI, I. Interface Adaptativa no ambiente AdaptWeb: navegação e apresentação adaptativa baseada no modelo do usuário. UFRGS, Porto Alegre, 2003.

NBR 9241-11. Requisitos Ergonômicos para Trabalho em Escritórios com Computadores, Parte 11 - Orientações sobre Usabilidade. ABNT. Rio de Janeiro, 2002.

NIELSEN, Jakob. Usability Engineering. Academic Press, Cambrige, MA. 1993.

PRATES, R.O., BARBOSA, S.D.J. Avaliação de Interfaces de Usuário - Conceitos e Métodos. Anais do XXIII Congresso Nacional da SBC. XXII Jornadas de Atualização em Informática. SBC’2003. Agosto de 2003. 